\title{
The Design of Social and Political Research
}

\author{
Philippe C. Schmitter ${ }^{1}$
}

Received: 12 June 2016/ Accepted: 2 September 2016/Published online: 20 October 2016

(C) Fudan University and Springer Science+Business Media Singapore 2016

\begin{abstract}
Good social and political research requires an explicit design. In this article, this is illustrated by a cycle of choices that begin with the transformation of an idea into a topic and end with the drawing of inferences from the patterns of association one finds. In between lie critical decisions about concepts, hypotheses, indicators, case selection, measurement and tests for association.
\end{abstract}

Keywords Selection of projects - Conceptualization - Hypothesis formation · Operationalization of variables - Case selection - Tests for association ·

Causal inference

\section{Introduction}

Let us begin with the assumption that you have had an idea that has led you to identify a topic that you believe to be of sufficient importance and of feasible execution so that you are considering conducting research on it. It may be a doctoral dissertation, a master's paper or just a simple seminar exercise, but regardless of length and complexity no topic can "research itself." You will have to translate itvia a series of strategic choices-into a project. It is this process of translation from something that you regard as problematic or puzzling into something that you can gather valid data on and make compelling inferences that constitutes your research design.

Granted, much social scientific research is not self-consciously designed, by which I mean, it is not subject to a deliberate and critical process of choosing its components and defending its overall configuration. In many areas of inquiry, the

Philippe C. Schmitter

schmitter36@gmail.com; philippe.schmitter@eui.eu

1 Department of Political and Social Sciences, European University Institute, Badia Fiesolana, 50016 San Domenico di Fiesole, FI, Italy 
design is literally given along with the topic. So much research has already been conducted on it that adding yet another case or extending it to cover yet another time period does not seem to require a novel effort of translation. Indeed, the universal desire of all sciences to produce cumulative knowledge seems to militate against continuously challenging and changing the standard way of doing research. If you do propose a change in design-say, a re-conceptualization of the topic, a revised instrument for measuring variation, a different way of selecting relevant cases, or a novel method for testing for association-you will risk confusing your reader cum critic. He or she may find it difficult to distinguish whether eventual differences in data or inference are "really" due to the topic itself or "merely" to your meddling with the established way of researching it.

Most young social scientists, however, will not be choosing topics whose research design is given. They will have to find or invent an apposite design-and they should be prepared to understand and defend the choices involved. Moreover, if their immediate or eventual intent is comparative-i.e. if they anticipate including more than one case or set of observations and drawing inferences across themthen, their choice of design will be even more crucial. Making the right strategic choices will greatly enhance the value of the data they gather and the inferences they can draw from them; neglecting these choices or taking them for granted could result in idiosyncratic scraps of information and inferences rooted in exceptional circumstances that make no reliable or cumulative contribution to scientific knowledge.

Figure 1 is a schematic and idealized representation of the complete "socialpolitical research cycle." Each of its boxes involves an important set of interrelated strategic choices, and its implication is that these should be made in the displayed sequence, i.e. beginning with an idea that defines a topic at 12 noon and proceeding clockwise until the researcher arrives at an evaluation of his or her findings that may or may not re-define the original topic at midnight. Inside the boxes lie a number of alternative courses of action. Choose among them wisely and you will do better research. Ignore them or fail to grasp their significance, and you run the risk of committing serious fallacies at each stage.

The most important message to keep in mind while proceeding through the entire cycle is to remember that there is no single best strategy or set of strategies for researching all topics. Everything depends on your point of departure, i.e. on the initial substance you have decided to research. At the beginning of the cycle in Fig. 1, the range of options tends to be most extensive-and, hence, most confusing. Interesting topics clamour for equal attention; different theories and concepts can seem equally compelling. As one proceeds clockwise, the successive choices are increasingly related to each other and the options become more limited. At some point, you may well adopt or fall into an "established disciplinary routine." You can save yourself a lot of time and worry by doing this-some would say that it is the inevitable and desirable product of "professionalization" in the social sciences-but this will only benefit you if your topic and, especially, its conceptualization is sufficiently isomorphic with the original, i.e. conforms to the basic characteristics of the topic that has already been successfully researched by others. Applying even the 


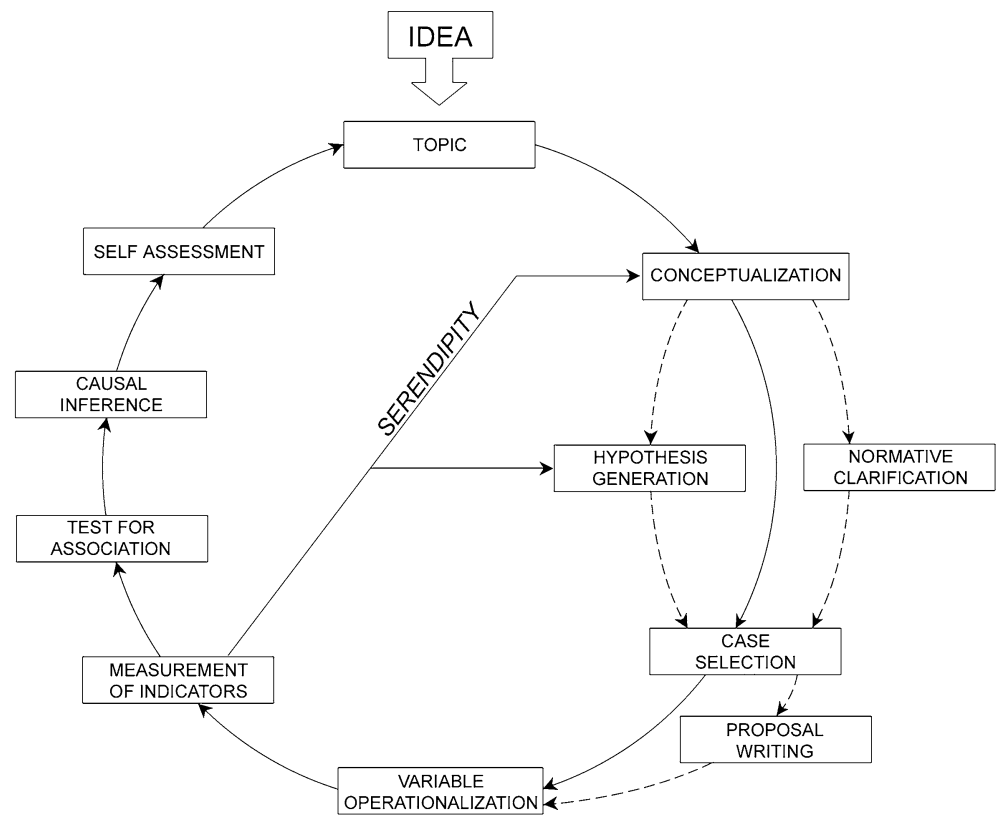

Fig. 1 The cycle of social and political research

best established and/or most fashionable design to the wrong topic can be a formula for disaster, especially, when it comes to drawing inferences.

Very few researchers "really" enter Fig. 1 at noon and leave at midnight. Most have consciously or unconsciously taken some shortcut to get started in the process. For example, many social scientists begin their research careers already knowing which case or cases they intend to work on. Not infrequently, it happens to be the country they come from or are trained in. The so-called "area specialists" usually have some prior personal commitment involving their knowledge of history, culture or language, and this tends to affect the topics they select. Others may have picked up some novel statistical technique or measurement device that they wish to show off-and they search about for an apposite topic to apply it to. Perhaps, the most common (and, in my view, pernicious) point of departure concerns theories or approaches that are currently fashionable in sociology or political science. Imbued with the conviction that only those espousing such a "paradigm" will find eventual employment, young researchers are prepared to take up any topic-no matter how trivial or obscure-if only to demonstrate their fidelity to its assumptions and postulates.

Do not presume that, once in the cycle, you will have to go all the way around. As we shall see in "Conclusion", there are many points of exit that will still permit you to make an original and significant contribution to knowledge.

Wherever you have "really" begun your research and whatever your motives for doing so, I recommend that you at least pretend that you are beginning at the top of 
Fig. 1-if only to help you clarify ex-post the design choices you should have taken deliberately or have already taken implicitly. Try to imagine that it all began with an idea about a substantive topic that is important to you and that only later did you place it in an explicitly conceptual context, elaborate specific hypotheses about its occurrence, choose the cases to study, and so forth.

Now, we can proceed to look sequentially into the "little black boxes" in Fig. 1. Attached to each of them the reader will also find a list of "possibly optimizing" choices and "potentially damaging" fallacies.

\section{Choice of Topic}

No one can predict where and when ideas will appear. With some knowledge of the researcher's personal and professional background, however, it may be a bit easier to predict the conditions under which an idea becomes a topic, i.e. when someone will attach sufficient importance to a given thought and place significant boundaries around it to make it worthy of investing his or her energy to explain how it came about or what its consequences might be. Needless to say, this highly personal effort at selection can be an important source of distortion throughout the rest of the design and, especially, when it comes to drawing inferences from whatever data distributions or associations are generated. The very fact that you care enough to select some topic probably means that you also value what it contributes or the effect that it has. However subliminal the thought may be, your values become embedded in the topic and can exert a persistent influence on your choices as you make your way around the rest of the research cycle. They may have an even greater impact when you decide to make a "premature" exit from the cycle.

It is also very often the case that one is attracted to a specific topic because the society or polity surrounding it also cares about it. Never is this more evident than when the subject matter is in crisis or in fashion. As social scientists, we are bound to be attracted to phenomena that call attention to themselves-whether they do so by creating further problems or by providing novel solutions, which is another way of saying that our topics tend to be either failed experiences at the end of their useful existence or recent successes that have yet to reveal their complete impact. Rarely does one come across designs explicitly focused on explaining the social or political phenomena that are mediocre or inconsequential.

Grosso modo, topics of research come in two guises: (1) projections where the researcher is confident that the existing approach and methods are adequate and deserve to be applied to units or time periods that have not already been covered or with greater precision to cases that only seem to be exceptional; and (2) puzzles where the researcher begins with the assumption that something is deficient in the way that the topic has been previously handled and that the units or time periods to be examined will demonstrate the existence of anomalies. Both projections and puzzles should be approached in the same "critically rational" manner, but the perspective of the researcher differs. If the topic selected is regarded as a projection, he or she has the intent (at least, initially) of confirming established wisdom and will take more seriously the obligation to make a cumulative contribution to knowledge 
within a specific discipline or paradigm. The perspective when tackling a puzzle leads one to seize on anomalies that seem to expose deficiencies in how the topic has been conceptualized, measured or reported, and that is more likely to lead the researcher to alternative concepts and methods-frequently, by drawing on other disciplines. Needless to say, both are capable of making valid contributions; both are needed by all social science disciplines.

\subsection{Possibly Optimizing Choices}

1. Choose a topic that you personally care enough about to be willing to spend the (always longer than expected) time to complete the project.

2. Choose a topic (and make an argument) that interests other social scientists (even those outside your field), and the better it is, the more will it interest those working in adjacent fields and disciplines.

3. Specify the temporal, spatial and, if necessary, cultural boundaries of the topic in a way that makes the research feasible, but does not make it trivial or "unique."

4. Acknowledge your initial source of inspiration for the topic and your personal preference about its outcome, without apologizing for them.

5. Never justify your selection only on the grounds that it has been "underexplored," and, especially, do not ignore, trivialize or dismiss what has already been written on the topic.

6. Try to reach as far back as possible in the corpus of social and political theory to find grounds for the relevance of your topic and, thereby, avoid being manipulated by present-day academic fad and fashion.

7. By all means, listen to your advisor and your peers, but be absolutely certain that, regardless of who first suggested it, the topic "belongs" to you.

\subsection{Potentially Dangerous Fallacies}

1. "Fad-ism:" Your topic (or method or theory) is being very much and very favourably discussed right now in your field, so that if you adopt it your work will be less criticized and you will be more likely to find a job.

2. "Wishful thinking:" Your topic has already produced well-publicized and promising results for the society or polity; therefore, if you conduct research on it, your findings will be taken more seriously and favourably.

3. "Ambulance-chasing:" Because the topic of your research is presently in crisis, you will have greater access to data and the public will be more interested in whatever you find out.

4. "Presentism:" Whatever you find associated with some topic in the present must have been there in the past and will probably remain there in the future.

5. "Standing on the shoulders of the past giants:" This will allow you to see further and to avoid being distracted by the squabbles among contemporary 
pygmies, provided those giants were looking at the same thing or, at the very least, in the same direction.

\section{Conceptualization}

Almost all substantive matters emerge "pre-conceptualized" in the strict sense that they can only be recognized by the potential researcher and shared with others if they are expressed in some intelligible language. The idea may come initially as a shape or a colour or an emotion, but words are the indispensable way in which it acquires factual specificity and shared significance. The complication for research resides in the high probability that the words initially involved will be those of the social or political actors involved-which implies that their words could bear many different meanings and be attached to a wide range of contrasting assumptions.

Conceptualizing a topic invariably involves translating the words that surround it in "real-existing" societies or polities into variables. These are not just fancy academic labels applied to a specific event or process. They should identify analogies, i.e. generic conditions that are shared by a distinctive set of events or processes and can take on different values over time-whether these are quantitatively or qualitatively observed. They acquire their peculiar status as causes or effects according to the way they are connected to other variables by theories. Once these variables have been assembled, whether from the same or varying theories, they constitute your provisional argument concerning the topic you have chosen to explain.

This brings us to "the Elephant-in-the-Room" that is so rarely mentioned, but so frequently the source of confusion at each stage of designing research. Even the most elementary and frequently used concepts-e.g. class, status, gender, age, region and religiosity for explaining voting behaviour-derive their meanings from being inserted into a more comprehensive (and presumably coherent) matrix of concepts. Their definitions may sound the same and, as we shall see later, operationalization of these variables may even be identical, but their role depends on prior assumptions and contingent relations that differ according to the theory, paradigm, approach or framework that is being applied. No single piece of research can possibly specify what these are. If you tried to do this, there would be no time or space left for your analysis. In other words, all social and political research is part and parcel of "the state of theory" prevailing at the moment it is conducted. No research can be conceptualized ex novo without reference to what has been produced already on that and related topics. This applies just as much to those who are trying to solve puzzles as those who are "merely" trying to make projections.

Choosing one's concepts is only the first step. Making them into variables means assigning a status to them and this is where their embeddedness in theory most saliently enters into the research design. The most important is to distinguish between those that are regarded as operative with regard to the chosen topic and those that are inoperative. The former are expected to play some discernible role in the explanation of outcomes-either as an explicanandum (i.e. that which does the 
explaining) or as an explicanans (i.e. that which is to be explained). The more elaborate the prior theory and, hence, the conceptualization derived from it, the more may it be possible to assign different statuses to the operative variables, for example, by distinguishing between primary and secondary ones, direct and intervening ones, continuous and episodic ones, and so forth. Needless to say, all these initially assigned roles can be inverted, especially where and when the objective is to explain a relatively long-term sequence of social or political processes. Inoperatives are variables that are present and can be expected to take on different values during the subject matter being researched, but whose effect is not expected to produce a discernible or significant difference. Needless to say, when it comes to making eventual inferences, allegedly inoperative variables may turn out to be an important potential source of spuriousness. Even constants, i.e. variables that were present but not thought to vary during the research period and, hence, a priori considered not capable of to contributing to variation in the outcome, may gain eventually in importance-especially when it becomes evident that the impact of operative variables was contingent on slight modifications or even simple reinterpretations of such background factors. Hopefully, irrelevant variables - those whose variation cannot conceivably be logically or empirically associated with the topic under investigation-will remain that way.

\subsection{Possibly Optimizing Choices}

1. As much as possible, avoid references to specific persons, countries or cultures with "uppercase" names by using only "lowercase" variables to describe them and their prospective effects in your argument.

2. There is nothing wrong with using a "hunch" as your starting point in conceptualization - the world surrounding most interesting topics is usually full of them-but try as soon as possible to identify the more generic theory in which this hunch is embedded, switch to its language, and explore its axioms or presumptions before going further.

3. Try to avoid "multi-collinearity"-clusters of variables that are closely associated with each other-and simplify by only using the dominant variable in such clusters or providing it with an ideal-type connotation.

4. Make as explicit as possible not only the operative, but also the inoperative variables and the constants in your argument-and be prepared to change their status in the course of conducting the research.

5. When using classification systems make sure that the categories are both inclusive of all observations and exclusive in their assignment of every single observation-and that all of them are potentially relevant to explaining outcomes, including those that are vacant for the moment.

6. Specify as soon and as explicitly as possible the universe to which your conceptualization is intended to apply in terms of both time and space.

7. Exercise caution when using concepts and variables across long periods of time or different cultural contexts since their meaning to actors and, hence, their effect may change. 
8. Strive for parsimony by eliminating double-dealing or superfluous variables, but without resorting to excessive simplification.

9. One way of doing this is to restate your argument several times and to make it more concise each time.

\subsection{Potentially Dangerous Fallacies}

1. “Obscurantism:” If you cloak your conceptualization in highly abstract terms or fit all of your observations into some complicated classification scheme, no one will notice that all you are doing is describing what happened.

2. "Attribute-ism:" The more definitional attributes or analogous properties you attach to a given concept, the more significant is it likely to be in explaining the outcomes you want to understand.

3. "Concept stretching:" A concept used successfully to identify an analogy among events in one time and place must be equally valid when applied to other times or places.

4. "Isolation:" Your preferred variable plays such an important role in explaining your topic that it can be conceptualized, measured and manipulated alone, without regard for the network of other variables surrounding it and the prior axioms upon which it rests.

5. "Novelty at any price:" Because existing concepts are so embedded in (old) theories, by inventing and using novel ones, you will be credited with greater originality in your research.

6. "Arbitrariness:" Since all concepts are basically arbitrary-a function of unpredictable practical uses and/or theoretical fashions-it will make no difference which ones you use, provided that your public and peers come to accept them.

7. "Consensual-ism:" If everyone in your discipline is using some concept and seems to agree on its meaning, as well as its explanatory relevance, you should feel safe to do so.

\section{Formation of Hypotheses}

Not all research designs involve the formation (or the eventual testing) of explicit hypotheses. There exists a very broad range of social and political topics for which it is possible to conceptualize the variables that may contribute to an explication, but not to assign any sort of provisional "IF ... THEN.." status to their relationships. For these topics, the apposite research logic is one of discovery and not of proof. The purpose is to improve one's conceptualization of a topic, probe its plausibility against a range of data and eventually generate hypotheses among its conclusions, but it would be premature to expect them as a pre-condition for conducting the research itself. 
Obviously, the determining factor is again that "Elephant-in-the-Room," i.e. the prevailing state of theory on a given topic. Substantive matters that are of recent occurrence, are only characteristic of a small number of cases, incite strong emotions or political controversies, or fall between different social science disciplines are obvious candidates for "discovery" status. The potential researcher is reminded that accepting this status should not be taken as a sign of inferiority. Somewhere behind all social scientific research that today routinely follows the logic of proof, there must have been a glorious moment in the past when someone launched a voyage of discovery. Unfortunately, behind the façade of increased professionalism and standardization of techniques, this message has been suppressed and only the most intrepid of young scholars will be likely to accept the challenge of trying to make sense out of alternative conceptualizations of the same topic or piecing together potentially coherent and general arguments by "process-tracing" on the basis of specific cases or admitting that, in instances of highly interdependent and complex social or political systems, it may never be possible to distinguish between independent and dependent variables, much less to express them in terms of a finite set of bi-variant relationships.

\subsection{Possibly Optimizing Choices}

1. Ensure that the assumption of any "IF ...THEN ..." relationship is sufficiently precise so that it specifies its "micro-foundation," i.e. the functional dependence, structural mechanism or intentional logic that is supposed to connect its variables and, where possible, introduce an independent measure of its presence.

2. Do not assume ex ante that only individual human beings are capable of laying "micro-foundations," when the "real-existing," i.e. historical, world is chock full of social and political units that have acquired the capacity to act collectively in ways that cannot be reduced to individual intentions and choices.

3. Ensure that the presumed cause is independent of the presumed effect, and not parallel or convergent manifestations of the same social or political process.

4. Where possible, specify explicitly the existence of intervening conditions or prevailing constants that must be always present for the hypothesized relation to produce its effect-even if these contextual factors do not vary during the research.

5. An ideal research situation can emerge when you find yourself in a "two-ring circus," i.e. when two rival versions of the same hypothesized relation are plausible and would explain diametrically different outcomes based on different theoretical assumptions.

6. Be prepared to recognize and deal with "equifinalities," i.e. similar outcomes that are produced by different sequences or mechanisms, when they emerge, and therefore to test different sets or, better, "strings" of hypotheses-not just isolated ones.

7. Remember that you always have three hypotheses to test, namely, the ones that suggest a positive or a negative relation, and the null hypothesis that no "IF 
...THEN ..." relationship exists. The latter should be regarded as the most probable in occurrence. Everything may be related to everything else in our complex environment, but not always in a predictable direction or to a significant degree.

8. Try ex ante when elaborating hypotheses to differentiate between variables that you think are "necessary" (always likely to be present when the outcome is present), "sufficient" (always and only present) and merely "helpful" (sometimes present; sometimes not). Never assume that your set of variables is going to be both "necessary and sufficient" and, therefore, make space for the inevitable "error term."

9. Since most research projects consist of "clusters" and "chains" of related hypotheses that contribute to explaining a selected outcome, it is often useful to draft a "model" of these simultaneous and sequential relations using time and space as coordinates.

\subsection{Potentially Dangerous Fallacies}

1. "Scientism:" If your variables are not organized into hypotheses with clearly differentiated independent and dependent variables, your research will not be scientific.

2. "Fear of failure:" If your hypothesis or hypotheses are disproved, you will have made no contribution to knowledge.

3. "Infinite regress:" All hypotheses about variable relationships in the social sciences are preceded by a potentially infinite historical chain of causality and consequence, therefore, it makes no difference when you choose to break into that chain.

\section{Selection of Cases}

For all but a few projects, the potential number of societies or polities affected by the chosen topic will exceed the researchers' capability for gathering data, testing for associations and drawing inferences. It is, therefore, normal that only some subset of these units will enter into your analysis. One of the most prominent of the strategic choices you will have to make involves the number and the identity of those to be included and the criteria you impose to select them. This can vary from one unit (the single case or person) to as many as are apposite (the universe of those affected), but there is a fairly un-escapable trade-off between the quantity of variables that have been included in your initial conception of the topic and the number of units for which you will be able to gather data. Including more cases probably also means poorer quality data, more missing observations and greater problems of conceptual equivalence. Inversely, the more narrowly you have defined 
and operationalized those variables, i.e. the lower they are on the ladder of abstraction, the less likely are they to be relevant in a wide range of cases.

Case selection may have its practical side when it comes to gathering data and, especially, making one's own detailed observations, but its real payoff is analytical. Manipulating the identity of cases provides most sociologists and political scientists with their closest equivalent to experimentation. It "simulates" the introduction of control variables. By "holding constant" across the sample such potentially relevant conditions as cultural identity, geographic location, level of development and temporal proximity, the researcher can at least pretend that variation in them is unlikely to have produced the outcome one is looking at. Granted that the controls can be a bit approximate and that there still will remain many potential sources of "contaminating" differentiation among units in the sample-still, this is the best design instrument that he or she has available. It should, therefore, be wielded with deliberation-and caution.

Strictly speaking, the researcher does not select individual cases but "configurations of variables" that co-habit the same unit and may even co-vary in a unique or distinctive fashion within that unit. But one cannot analyse "France" as such and compare it with, say, "Spain" or "Italy." There are simply too many different (and potentially relevant) conditions within each of these countries with regard to almost any topic you chose to work on. This holds even when comparing micro-units within the same country where the number of variables can be more reliably controlled due to common constraints at the nation state level. The so-called "wholistic" research is, therefore, largely an illusion in social and political research and, when tried, usually amounts to little more than a detailed or "thick" description of one case (or of parallel ones if more units are covered).

This is not to say that there are not significant differences between designs that are driven by the effort to isolate a small number of variables and test exclusively for their association with other variables across a larger number of units, and designs that begin with a large number of interrelated variables (often combined via ideal type constructs) within one country and then seek to find significant and persistent connections across a few, carefully selected, units of an allegedly comparable nature. But in either strategy, what you are usually comparing are variables - one or many, alone or in clusters - not units. One exception concerns some research in international relations where the underlying theory (often erroneously) makes the assumption that the only relevant actors are sovereign states acting in their unitary national interests.

This brings us to the second aspect of case selection that has long been taken for granted and yet has recently become of growing concern. For a unit of observation to be a valid case for analysis, it must possess identical or, at least, comparable degrees of freedom with regard to the topic under investigation. A design that drew inferences-descriptive or causal-from a sample of units composed of Brazilian municipalities, Mongolian provinces, Spanish estados autonómicos and the permanent members of the United Nations Security Council about the efficacy of taxation systems would not attract much attention. Much as its author might (correctly) protest that this "sample' embodies a "most different systems design," 
critics would (rightly) object that actors in these units did not have remotely equivalent powers to make or enforce their decisions about taxation.

The usual formula for getting around this problem was to select only units that were at the same level of aggregation and enjoyed the same formal status within the world social and political system. This presumably explains why so many comparative research projects have been based on nation state units or, to a lesser degree, on relatively autonomous sub-national units within federal or confederal systems. The reductio ad absurdum of this strategy has been reached with large $\mathrm{N}$ comparisons containing all the members of the UN for which data can be obtained-despite the blatant fact that these so-called sovereign states have radically divergence capabilities for governing their respective populations or even satisfying their most elementary needs.

Since Donatella della Porta has contributed an entire chapter to this volume that deals extensively with the issues involved in case selection, I have little more to add. I will, however, insert a pedagogic device that I have found useful in explaining to students what their options are at this point in the research cycle (Fig. 2).

Assuming that all researchers are committed to producing scientific knowledge, the preferred case selection strategy should usually be the experimental one, i.e. choosing the units of observation randomly and introducing some element of change in a subset of them while holding variation constant for the others. Unfortunately, most social and political scientists have to operate in "real-existing" settings where this is not possible. Even when they are permitted to engage in experimentation, the topics tend to be so trivial and the settings so artificial that projecting inferences based on such findings to more "realistic" contexts are very hazardous.

Quasi-experiments may be second best, but they offer some interesting advantages, both with regard to the efficiency of research and to the credibility of inferences. The case base can be as low as one, although it is better to replicate the quasi-experiment in several other settings, if possible, within the same time frame. They are, however, limited to real world situations where the independent variable is highly discrete and temporally circumscribed and where data-gathering over a sufficient period of time has been consistent and reliable. Assessing the effect of a new public policy or the impact of some unexpected social or natural event tends to fit this narrow bill of particulars, but only if nothing else is happening to the unit or units at the same time. This is also a strategy of case selection that is especially vulnerable to diffusion or contagion effects, if the units involved know of each other's behaviour.

Most sociologists and political scientists will have to settle for the study of variations in their subject matter that appear 'naturally', whether within a single case or across different numbers of them. The chapter by Della Porta explores the implications of making these choices.

There are, I would add, a number of other alternative strategies that are not usually included in texts on research methods or design-presumably because their scientific status is dubious. They typically arise in contexts in which it is risky or impossible to observe and record the behaviour of "real-existing" social or political units. All involve what Max Weber once called "thought experiments." The best known goes under the rubrique of counter-factualism and involves the researcher in 
DESIRE TO PRODUCE

SCIENTIFIC KNOWLEDGE

ABOUT SOCIETY/POLITY

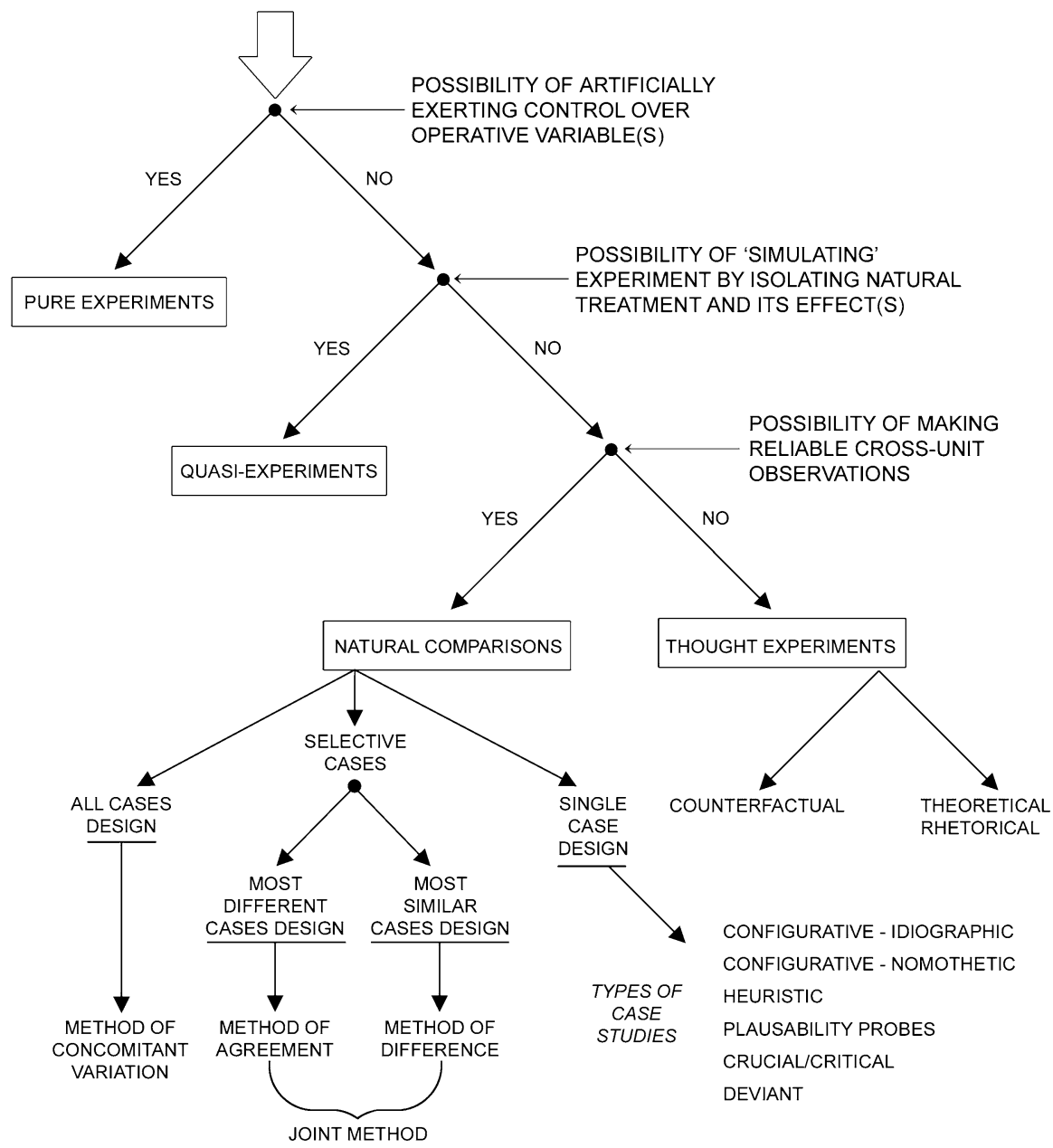

Fig. 2 A schematic for the selection of cases

an effort to imagine what would have happened to the topic if some condition, person, event or process had not been present. Usually, this focuses on a single country-e.g. How would Germany have evolved politically if Hitler had not been "available" in the early 1930s? - but it can also be applied to a sample or even the universe of cases-e.g. What would be today the level of international insecurity in Europe if the EU did not exist? Or, how many people in the world would know how to speak English if the Americans had lost their Revolutionary War? If this sounds "exotic" and a bit "flaky," you should remember that every time that you invoke 
the famous and indispensable Latin phrase, ceteris paribus, before advancing a hypothesis, you are guilty of being a counter-factualist.

Moving even further from social scientific orthodoxy, one finds a vast number of seminar exercises, MA papers and $\mathrm{PhD}$ dissertations that are essentially rhetorical, theoretical or normative. These certainly deal with topics-often more important ones for "real-existing" societies and polities than those chosen by empiricists cum positivists - but their purpose is to follow the development of concepts or discourses over time, or to examine the logical consistency of particular arguments, or to promote the ethical acceptance of specific forms of human behaviour. Should we conclude that these projects do not involve research-even though it is not uncommon that works of this sort have a "heuristic" and "nomothetic" way of influencing eventual empirical inquiry? Just think of the impact of recent works by John Rawls, Jürgen Habermas and Jon Elster upon how even the most "hardcore" empiricists select and conceptualize a wide range of topics.

\subsection{Possibly Optimizing Choices}

1. If you are not trying to cover the entire universe, consider the possibility of selecting a sample of cases randomly and debate with yourself how that would be likely to affect your project.

2. As a rule, when randomization is excluded and you have to be purposively selective, choose your cases based on their relation to the independent variable or variables rather than the dependent variable or variables.

3. When you make this choice, try to ensure that the cases chosen 'represent' as wide a range as possible of scores on those independent variables.

4. When your topic will not permit this, i.e. when you are motivated to research something precisely because it involves a compelling, arresting or extreme outcome, and you therefore have to select on the dependent variable, remember this as a potential source of bias when it comes to drawing inferences.

5. Keep in mind that you do not have to use only one strategy of case selection and that so-called "Nested Strategies," i.e. where you start with a large N of cases and relatively few crudely measured variables and, subsequently, shift to a small $\mathrm{N}$ with a much more detailed "battery" of variables-can give you the advantages of both strategies when it comes to drawing inferences.

6. Always prefer the lowest level of spatial or functional aggregation that is compatible with the actor behaviour presumed by your conceptualization, since you can subsequently re-assemble your research upwards-but not downwards-in scale.

7. No matter which or how many cases you initially select, some may prove to be "decomposable," i.e. you may be able to generate additional cases by dividing up the initial ones, but only provided these subunits possess some and the same degree of autonomy. 
8. Before selecting the number and identity of the cases for which you intend to gather data, make sure that you are aware of the system that you originally used for classifying your topic and ask first: "What is this a case of?" Only after satisfying that demand, will you know what units are "eligible" for inclusion and you can proceed to exclude some of them for good reason.

\subsection{Potentially Dangerous Fallacies}

1. "Notoriety:" Just because a given case has been prominent in public discussion, it will be more interesting to research and that others will pay more attention to your research.

2. "Numbers:" It is always advantageous to have a larger number of cases, even when, by adding them, you are compelled to attenuate their relation to the topic or to use less valid indicators.

3. "Cruciality:" Because a given unit is an outlier according to your criteria of case selection, it will be a crucial case whose conformity or non-conformity provides a definitively significant test of causal association.

4. "The illusion of control:" Selecting cases because they seem to share certain general cultural, locational or structural characteristics necessarily controls for their relevance-despite the possible persistence of more qualitative sources of variation that might be affecting variation.

5. "Contemporaneousness:" In units chosen for comparison within the same time frame, the actors must have similar (or, at least sufficient) awareness of the relevance of common variables and be capable of acting upon them simultaneously.

6. "Imitation:" When actors in the selected units are acutely aware of having to deal with some topic within the same time frame, they will also be sensitive to what others are doing about it and will learn from each other's successes and failures.

\section{Writing of Proposal}

This stage in the research cycle is "optional," although highly desirable in my opinion. Different graduate programs place greatly different emphasis on the importance of defending a formal proposal before one's research committee, faculty colleagues, student peers or all of the above. Some MA and $\mathrm{PhD}$ advisors are keen on requiring it before allowing the candidate to "go into the field." My personal experience suggests that the greater the plurality of approaches or paradigms surrounding a given topic and present in a particular institution, the greater will be the emphasis on writing and defending your proposal. In scholastic contexts dominated by a single theoretical or disciplinary orientation, the effort may be eschewed completely. The reigning orthodoxy favours problems rather than puzzles 
and may even dictate in considerable detail how topics should be conceptualized and operationalized. At the extreme, there is no "field" to go to, no specific cases to select and no measurement details to discuss. What matters at this stage is the normative or logical consistency of the "argument," i.e. of one's conceptualization of the topic, and how well it conforms to prevailing orthodoxy. The number and identity of cases are relatively unimportant, if not irrelevant, to the extent that both prior axioms and subsequent expectations are believed to be universal. The data can be simulated or assembled from the usual sources for illustrative purposes. The eventual inferences are usually predictable and in line with original expectations. The fellow members of your "research club" will enthusiastically congratulate you on your cumulative contribution to knowledge. Practitioners of other disciplines and members of other clubs within your discipline will yawn and tell you that you have "re-discovered the wheel" or produced something utterly trivial. In other words, there are costs as well as benefits in belonging to an established research tradition.

Another condition affecting the utility of proposal writing is its potentially critical role in obtaining research fundings. Where such support is assured or not subject to competitive pressures; the researcher may content him- or herself with a brief statement of intention. Otherwise, your ability to summarize coherently and justify convincingly the design choices that you have made up to this point could make all of the difference in determining whether you will be able to carry out your project at all. Although it is not frequently discussed openly, this 'commercial' aspect of proposal writing can also be a source of distortion when the preferences of the sponsor come to be anticipated in the proposal itself and the researcher finds him- or herself pandering to them by modifying the topic, changing its conceptualization, restricting the range of hypotheses and even selecting different cases in an effort to please the prospective sponsor. Needless to say, more experienced researchers soon learn how to "fine-tune" their proposals to get support from donors and then go on to follow the course of inquiry they think will lead to the most compelling inferences. Fortunately, national or supra-national sponsors rarely control for conformity between proposals and the research actually carried out. At most, they may be interested whether or not the policy implications drawn from such research conform to their preferences.

The 'real' purpose of writing a proposal should be give the researcher a chance to sit back and reflect critically on the strategic choices he or she has made-and to exchange these reflections with supervisors and peers before plunging into the inevitably messy and absorbing process of gathering data and trying to make sense out of them. There may be subsequent moments for self-criticism and changes-see the remarks below on the importance of serendipity-but writing and defending a proposal at this stage offers a unique opportunity to "re-write" and "re-submit" before becoming irrevocably locked into a course of action.

Given its "optional" nature, I have placed all of my more specific suggestions on this issue in an "Appendix" at the end of this chapter. It offers my version of what "the ideal research proposal" should contain. 


\section{Operationalization of variables}

In principle, the conceptualization of variables should be carried out beforehand and without regard for how they will be converted into indicators and eventually measured. There is a very good reason for this. What is of paramount theoretical importance is to specify clearly the condition or factor that is supposed be present to produce some anticipated effect-alone or in conjunction with other variables. Having previously and independently conceptualized the projection or puzzle in such a fashion should provide a strong incentive subsequently to specify the observations that need to be made to verify the presence, magnitude, direction or persistence of that variable. During the early stages of research, this means that you should adopt the attitude that all social and political variables can potentially be operationalized - and later be prepared to compromise when you start looking for indicators in the real world.

In practice, unfortunately, anticipations of such difficulties do tend to intrude and can even inhibit scholars from using concepts that are known to be "impossible" to operationalize. Just think of such indispensable political properties as power, authority and legitimacy, or of such social ones as esteem, respect and trust. For none of them is there a standard and easily accessible set of measures. Even elaborate (and expensive) attempts to operationalize them based on "reputational" criteria from public opinion surveys have been problematic. Criticisms of these efforts become more insistent the more such indicators are stretched across countries and over time.

Another way of putting this dilemma is that there are bound to be trade-offs that have to be made at this stage in the research cycle. The higher one's concepts are on the ladder of abstraction-and, presumably, the wider their prospective range of application - the more difficult will it be to make convincing observations about their presence in a specific case or set of cases. Increase the number of units in your study - either of persons or organizations - and you are almost bound to run into problems with missing data and misleading indicators. Do not be afraid to make these trade-offs, but do so self-consciously. Tell your reader cum critic when you are settling for a less satisfactory indicator or a less specific level of observation. Be prepared when necessary even to eliminate cases, but also be sensitive to how this may distort your eventual capacity to draw inferences. Those research sites where operational requirements are most difficult to satisfy are usually places where social and political behaviour is the least "normal" and their exclusion from the design will probably narrow the range of variation and reduce the eventual strength of association.

The theme that haunts all aspects of this stage of the research cycle is validity. Do the observations you propose to make accurately reflect and, hence, capture the meaning of the concepts you have chosen to bear the burden of explanation? No matter how accurate the observations, how comparable they are across units, how replicable they turn out to be when another scholar makes them, if they are not valid 
your research will have broken down at one of its most vulnerable points. You may well have discovered something important and the associations revealed by your indicators might be incontrovertible, but you have not proved (or disproved) what you started out with. Your findings are irrelevant in the strictest sense. They have told you nothing about the topic you announced that you intended to work onunless you are prepared to rely on serendipity and re-conceptualize your entire project from its very origins.

\subsection{Possibly Optimizing Choices}

1. Pay close and critical attention to the correspondence between your initial concepts and their proposed indicators or assessments by comparing them to research by others on the same or related topics.

2. Be especially wary of variable specifications and, most particularly, of empirical indicators that have been applied routinely over time and across units to measure different concepts.

3. Make sure that the concept and its indicator(s) are applied to the same level of analysis and are as close as possible in level of abstraction.

4. When available, use alternative operationalizations and multiple potential indicators and, where necessary, rely on 'triangulation' among them to resolve disparities and to improve validity.

5. All things being equal (and they never are), you are better off using unobtrusive rather than obtrusive indicators, since the actors whose behaviour is being observed will have less of an opportunity to respond strategically, ethically or emotionally to your request for information.

6. Remember that there are various ways of assessing the validity of indicators, ranging from consensus among independent respondents to co-variation between different 'internal' measures and, least reliably, correlation with other hypothesized 'external' outcomes.

\subsection{Potentially Dangerous Fallacies}

1. "Availability:" This indicator exists and has been used successfully by others; therefore, it must be valid when applied to your topic.

2. "Operationalism:" You decide only to include in your analysis variables for which you know that a valid (or consensually accepted) indicator already exists.

3. "Mimitism:" ' $X$ ' got away with using data on this to indicate a concept similar to yours, even when drawing upon a different theory; therefore, you can safely use it for the same purpose.

4. "Ignorance of the uncertainty principle:" If you operationalize a variable by intruding on the "real-existing" world of your respondent, you can nonetheless ignore the possibility that his or her answer will be contaminated by prevailing 
norms of correctness or strategic calculations of interest, or that you will be creating rather than measuring variation.

\section{Measurement}

At this point in the cycle, your choices will be more or less dictated by the ones you have already made-whether you did so consciously in relation to the specificity of your problem or puzzle (as I hope was the case) or whether you settled into an established research tradition-whether quantitative or qualitative - and obediently followed its dictates. Moreover, there is a good reason why you should let yourself "go with the flow" at this point. Using existing techniques of observation and indicators for variables not only saves you a lot of time and anxiety, but can also provide you with an element of internal "quality control" - provided, of course, that the measures used are valid, i.e. capture the characteristics of the variable that you are relying upon for an eventual explanation. When it occurs, successful replication of previous research is a very desirable result-and one that can be personally very reassuring. Should you decide to invent and apply a new indicator or, worse, battery of indicators-especially to measure some frequently used variable-you will have to make an especially strong effort at justification. Otherwise, you will run the risk at the inference stage of confounding the reader: Is this seemingly compelling finding really novel or is it only due to some change in measurement?

The discussion on measurement tends to be dominated by the distinction between quantitative and qualitative indicators - with a marked bias in favour of the former. There is no reason to be surprised by this since most methods texts are written by quantifiers and they have convincing arguments in their favour. Numerical data are said to be more reliable, i.e. more likely to provide agreement among independent observers, more accurate, i.e. more likely to produce agreement across units, and more useful, i.e. more compatible with different ways of testing for association. Certainly, the social science disciplines have tended to assign greater "scientific status" to quantitative over qualitative research-and to reward its practitioners accordingly. In the pecking order among such disciplines, there is an almost perfect correlation between status (and salary) in academe and the use of numbers and equations.

This is unfortunate for at least three good reasons: (1) it has encouraged researchers to attach numbers to variables when the validity of their connection with the designated concept was dubious; (2) it has resulted in the exploitation of standard numerical indicators whose multiple components are often theoretically disputable and whose weighted combinations are poorly understood by those who use them; and, (3) it has discouraged the innovative use of more direct and imaginative techniques of observation-precisely to capture qualities inherent in complex and contingent relations. True, you can assign a number to anyone and anything, but nothing guarantees that the assignment will produce relevant information. If these qualities are differences in kind (nominal) rather than in magnitude (cardinal or ordinal), then - whatever the rule of their assignment- the 
number could well be a worthless piece of dis-information. What matters is how you have conceptualized your topic, not the allegedly superior virtues of one over another form of measurement.

Of all of the stages in the cycle, this is probably the one that is best suited for serendipity, i.e. for learning from the research process itself in ways that can feedback to your previous choices and lead you to introduce improvements in them before "path dependence" has completely taken over. At last, you are back in touch with the "real-existing" subjects/agents of your topic-having spent much time wandering around making abstract "disciplinary" decisions. If you are lucky, they will talk to you directly about their intentions and perceptions, and they may even have some opinions about what you asking them and intend to do with their answers. Even if your research relies exclusively on secondary or publicly available sources, there can be "voices" in such documents that can speak in ways you have not anticipated. Of course, there will be a lot of sheer "noise" generated by the data you are gathering and that can be very confusing when juxtaposed to the relatively (and necessarily) parsimonious approach you have been applying to the topic. Nevertheless, keep your eyes, ears and mind open for subtleties and surprises, and be amenable to introducing "course corrections"- even some that go all the way back to the boundaries you initially placed around the topic or key aspects of your original argument.

\subsection{Potentially Optimizing Choices}

1. Routinely test for the reliability of indicators, if possible by using alternative sources of data and/or alternative persons to score the data.

2. If validity requirements can be satisfied, opt for quantitative over qualitative measurement since the technical advantages are considerable and because you can more easily move from the former to the latter.

3. Always opt for the highest, most informative level of measurement possible (given the nature of the variable), since it will later be possible to shift to a lower level. Cardinal data can always be made ordinal and virtually anything can later be dichotomized or filed away in nominal categories-but you cannot move in the inverse direction.

4. Make your instructions-even if only for your own use-concerning the assignment of quantitative scores or qualitative labels as transparent and complete as possible so that the measurement operations can be replicated by you or someone else in the future.

5. Especially when working on the macro-level of a complex society or polity, most variables will contain multiple components and be indicated by composite measures-which should obligate the researcher to devote concerted attention to how such "scales" are aggregated.

6. Especially when gathering information over time about social or political processes, make sure to check that changes are not due to modifications of the instruments of observation rather than to changes in actual behaviour. 
7. Many measurement devices are calibrated in such a way that they only pick up relatively large-scale and consequential changes in variables, which means that they may systematically fail to capture more modest and gradual ones. Social and political 'revolutions' are always recognized; 'reforms' are more often underreported-until their effects have accumulated sufficiently to draw attention to them.

8. Try to estimate before actually gathering the data where the error sources are most likely to come from and how they will affect your findings. Worry less about random errors (they will attenuate possible associations) than about systemic ones (they will bias the direction of your findings).

9. Of course, try to catch yourself before adjusting the data or correcting for errors in it in ways that make these data fit better the general expectations or specific hypotheses you started with.

\subsection{Possibly Dangerous Fallacies}

1. "Composite-ness:" Many concepts are complex and multi-dimensional in nature and, therefore, can only be measured by similarly complex and multidimensional indicators-regardless of variation in their internal structures and, hence, the probability that identical scores will be assigned to quite different clusters of variation.

2. "Longevity:" It is always better to use an indicator that has been around for some time, used in a variety of research settings and can provide the researcher with a longer time perspective-despite the likelihood that during this period the techniques for measurement will have changed and the meanings of items for actors may not be the same.

3. "Clarity:" It is always preferably that each variable be given a specific and unambiguous score-even if the nature of its conceptualization and theoretical status is calculatedly "fuzzy" or "radial."

4. "Reification:" What you are measuring is identical to what you have conceptualized which, in turn, is identical to the way in which actors perceive "it"-regardless of how much is lost in translation as the researcher moves from one realm to another.

\section{Test for Association}

By now, the researcher may have momentarily lost almost all strategic control over his or her project and, at best, should consult one among many texts on methodology to discover which among all of the verbal or mathematical, symbolic or numerical, parametric or non-parametric, deterministic or probabilistic devices that are available for testing for association best fits the data that he or she has gathered. 
Variables can be associated with each other in different ways. Typically, the social scientist will be interested in direction or whether the fit is positive, negative or null; strength or how much one variable affects another; and significance or the likelihood that the fit could simply have been due to chance. Since his or her research will almost inevitably be "historical," the time, timing and sequence of how they fit to each other should also be important-indeed, these chronological dimensions often provide the basic orientation to how one's findings are presented and defended.

The reason for this is that the most powerful means of testing for the fit among variables and, therefore, for presenting one's findings has long been to tell a believable story in chronological order. And there is no reason to believe that this is not still the case. Perhaps, within some highly professionalized niches in sociology and political science, story-telling is no longer regarded as acceptable. The occupants of these niches-not infrequently, Americans or those trained in America-have forgotten that their disciplines are profoundly and irrevocably historical. What counts is not just what happens, but when it does and in relation to what else has already happened or is simultaneously happening. Moreover, the actors themselves are not just passive recipients of scores, but active and reflexive keepers of the score. They remember what they and their ancestors did in the past and their preferences in the present are conditioned by this knowledge. In my opinion, no means of testing for such associations has yet been invented that can supplant or even surpass the chronological narrative in capturing these subtleties of time and timing, and in bringing simultaneously into focus the multitude of variables involved in the sheer complexity of most social \& political phenomena. The narration of your findings can, no doubt, be considerably bolstered in credibility by inserting quantitative tests about specific associations into the basic narrative. Cross-tabulations, rank-orderings, regression equations, factor- or small-space analyses, even mathematical models, can often be helpful, but primarily when analysing topics that are heavily circumscribed in time and space and that can be separated into relative simple and repetitive components.

Even social and political scientists firmly entrenched in their respective niches and relying exclusively on quantitative data may find it occasionally useful to come up with a plausible story that places the associations they calculate and the inferences they draw in some chronological order. Narration can also serve to fill in the gaps between cause and effect by providing a verbal description of the mechanisms involved-especially when mathematic formulae and formal models typically treat such exchanges as taking place within impenetrable "black boxes." Hardcore quantifiers may scorn this as unscientific "babbling," but it may offer them their only outlet to a wider audience.

This explains why these more specialized "niche players" are often researchers whose findings circulate only among small groups of conoscenti and are utterly incomprehensible to outsiders. Presumably, there are those who regard such incomprehension as proof of their success as scientists, but whenever sociologists or politicologues aspire to enlighten and influence wider publics, they will either have to learn how to narrate their findings or to hire someone else to translate the esoteric results of their tests into more intelligible stories. 


\subsection{Potentially Optimizing Choices}

1. Never forget the "Inter-Ocular Impact Test" that consists in simply eyeballing the data-scatter plots are especially useful for this-and forming your own visual impression of what is going on among the variables and across the cases.

2. In other words, always try to apply different tests of fit and only try the more demanding ones once you have experimented with simpler ones-even if you conclude by using only the one that is considered "cutting-edge" with regard to the data you have assembled.

3. If possible (and it will be much more possible with quantitative designs), manipulate the number and identity of cases in the sample or universe to test for the robustness of initial results_-and do not be discouraged if this shakes up their fit, but try to discover what implicit control variables may have been injected to produce such different results.

4. Remember that most tests for association-quantitative for sure, qualitative for some-are exceedingly sensitive to extreme cases, so that you may be well advised to eliminate them in order to find out how persistent or significant is the association among variables when only more 'normal' units are included in the analysis.

5. Also, remind yourself of the time dimension and test whether successive cross sections through the data-say, at 10-year intervals-produce equally strong associations. If they do not, reflect on what intervening or contextual variables might be responsible for the new findings.

6. Your tests for association will be all the more convincing, the more effort you put into falsifying initial hypotheses, rather than merely seeming to verify them by grasping at all favourable distributions of data.

7. The treatment of 'deviant' cases that do not fit the general pattern of association is often taken as an indicator of how seriously the researcher accepts the task of falsification. Ignoring them (or transforming their scores) suggests that you are excessively concerned with verification; embrace them, exploit their contrariness, try to determine the extent to which they call into question the hypothesis and you will gain favour as a "falsificationist."

\subsection{Possibly Dangerous Fallacies}

1. "Spuriousness:" You have found a close association between two variables and you report this finding-without considering that if you were to introduce a third variable, it might explain variation in both of the original ones.

2. "Contingency:" The associations you find are strong and significant, but only if and when certain, usually un-specified, contextual variables are present.

3. "Curve-fitting:" Since there is always "noise" and "error" in the data, it is permissible to "smooth" distributions by transforming the raw data or eliminating outliers and this will usually result in a more "satisfactory" fit. 
4. "Anachronism:" Whatever the associations that satisfy your test criteria and the time period covered by your research, the findings they generate will be valid whenever.

5. “Ad-hocracy:" At some level of abstraction and measurement, each case can be uniquely identified and used to "explain away" any and all observed deviations from the outcome predicted by those variables included in the study.

\section{Causal Inference}

This is by far the most hazardous - and the most rewarding - of the stages in the research cycle. It is the one in which you will have the least disciplinary or academic guidance and, hence, the widest range of discretionary choices to make.

Many social researchers will have exited the process before arriving here. They will have made their accurate observations, published their empirical descriptions and gone home. Others will have stopped even earlier, before having gathered any data, and left satisfied that they have advanced further the plausibility of an argument or helped to specify the universe to which it can be applied. Some will have gone further and proffered tests-numerical and narrative-illustrating how frequently and strongly variables have been associated with each other. But they will have prudently refrained from trying to answer the two obvious questions that the complete research cycle is supposed to address: (1) the retrospective one of why and how did these variables combine to produce the outcome that was the topic of the research in the first place; and (2) the prospective one of what will be the consequences of this in the future and when will these consequences happen.

Consider, as an example, the current controversies over climate research. Do you think that, if climatologists and other scientists had merely filed reports demonstrating that temperatures have been rising across the planet and that various chemical substances have been accumulating in its atmosphere, there would have been much of a reaction? As far as I know, these facts have been accepted by all as uncontroversial. It was only when these researchers correlated these indicators and drew the inference that increases in them masked a causal relation that could not due to chance or fate that things began to get stirred up. And when they attributed primary causation to factors related to human intervention and, even more, when they began to advance threatening projections about what will happen in the future, then, all hell broke out!

Without even hinting that all social scientists have a responsibility for generating such controversy, they should feel a more modest responsibility for exploiting their data to the fullest extent possible and that almost inevitably commits them to drawing retrospective and (sometimes) prospective inferences. Just think back to the number of occasions when you have read a report on extensive and expensive research and still found yourself asking the "why" and "how" question at the end. Granted that this could be regarded as favourable by younger researchers since it means that there is a very considerable amount of unexploited data out there just waiting for "secondary analysis" at low cost. Nevertheless, it is lamentable when 
the scholars who initially chose the topic, conceptualized it, selected the cases, and gathered the data do not go as far as they could in drawing "grounded" inferences about the causality it might reveal. Manuals for sociology and political science are full of sage advice concerning the limits of doing this. Not infrequently, teachers of graduate courses and dissertation advisors will revel in providing the student with egregious examples of researchers who exceeded the confines of their data or ignored the contribution of other variables, and made what proved to be erroneous statements about causality or consequence.

The controversy that tends to dog most discussions about inference is generalizability. A cautious researcher who draws inferences from his or her findings that are restricted to the cases investigated and the time-period covered is less likely to face much criticism-or to generate much attention from others. Specialists on the topic will, no doubt, have something to say about the validity of indicators, the accuracy of measurements and the appropriateness of tests for association-but it is not until you dare to generalize across temporal, spatial or cultural contexts, i.e. until you trample on some else's turf, that you will be seriously challenged. No one likes to be told that his or her topic can be differently explained by someone intervening from another theoretical or disciplinary perspective.

There are good reasons for this. Although they may seem arbitrary or anachronistic (and some no doubt are), the lines of specialization built into different social science disciplines have served to enforce professional standards and preside over the accumulation of knowledge. Generalizations that are based on alternative conceptualizations and/or novel methods should be especially carefully scrutinized. Nevertheless, this is where the real scholarly excitement lies-this is where "seminal" contributions are to be made-provided the researcher is well prepared to face his or her critics.

Needless to say, the strategy of case selection will play an especially significant role. Single case studies are rarely convincing-even the so-called "crucial" ones. Large $\mathrm{N}$ studies should be less objectionable, were it not for the fact that many of their cases are dubious in terms of their (alleged) common capacity to act and the probability that behind any associations found in the whole universe there are bound to be subsets of cases (regions?) where the fit differs considerably-and may even reverse itself. Middle-size samples based on controlling for the "usual suspects" (geographic location, development, size, religion, cultural area) by their very nature inhibit generalization, unless they are replicated for different samples. Indeed, replication can be a powerful weapon-and not just to the extent that other sets of cases or periods of time produce the same direction, magnitude and significance of association. If you can show that a reliable pattern holds at different levels of aggregation within the same sample, you will have added considerable compulsion to the inference that it is more likely to hold elsewhere.

The other critical factor will come from accusations of researcher bias, often alleged to be the product the national or disciplinary context in which the researcher operates. It is only human to prefer to discover what you thought was there in the first place and, then, to extend that finding to other places you know less about. Most often this can be attributed to a natural tendency to "overobserve" what you 
expected to see and to "underobserve" variation that you were less prepared to encounter. To this "Type One Confirmation Bias," one has to mention that Type Two Errors also exist. In this instance, for some perverse reason, the researcher prefers to reject his or her original hypothesis and, thereby, underestimates the degree of association that actually exists. Whether the peculiarities of national cultures or academic disciplines have any thing to do with either of these typical errors seems dubious to me, but there is no doubt that both exist.

The most secure way of guaranteeing enduring respect for the inferences you have drawn from your research-and of securing your place in the Pantheon of Notable Social Scientists - is to place them under the protection of a "Covering Law." This law offers an explanation for a much broader range of social or political phenomena, e.g. the Darwinian "Law of the Fittest." It should be widely, if not universally, accepted by the Notables who have preceded you and, ideally, it should not be derived from the theory you started with. But do not worry if you do not make it to the Pantheon. Your contribution to knowledge can still be significant and your career as a social scientist still very rewarding.

\subsection{Possibly Optimizing Choices}

1. Add alternative explanatory variables suggested by other cases or experiences (if available without conducting an entirely new piece of research) to discover whether the original fit within your sample holds up.

2. Probe your data by subtracting sub-sets of cases in the initial sample to see how robust the findings based on it were, especially when you think you are dealing with the entire universe but have reason to suspect 'regional' variations.

3. Be careful not to "anchor" your inferences by relying too much on a single prominent association among variables at the expense of lesser (and less expected) ones.

4. When assembling a batch of inferences from a research project, do not privilege or attach greater significance to findings that were easier to document or closer to your own experience.

5. It will be risky, but try on the basis of your inferences from a given sample to predict what analogous behaviours have been in a different sample of persons or places that you know nothing about.

6. Even more risky, apply the inferences you have draw to predicting the future performance of the units you have studied.

\subsection{Potentially Dangerous Fallacies}

1. "Triumphalism:" You have discovered a significant finding; therefore, your work is over-even though it could be the result of some variable you forgot to include and that may be very prominent in other cases or samples. 
2. "Pago-Pago-ism:" Whenever you think you have found something that applies everywhere, there will always be some place that you do not know (or not even have heard of) where the finding does not fit-and there will always be a scholar who knows the place and will inform you of your error.

3. "Exceptionalism:" You chose to study a particular topic only in a particular country because you considered that the context was exceptional and, then, you turn around claim that the findings you have observed are universal.

4. "Replicability:" Associations among variables that have been found to be consistent in direction, strong in magnitude and significant at one level of analysis will replicate themselves at other-lower or higher-levels of aggregation within the same sample.

5. "Cognitive dissidence:" If variables that simply "should" not go together still seem to be associated, this must be due either to some unidentified measurement error or conceptual confusion so that you are justified when drawing any inference by excluding the case or withdrawing the variable from your analysis.

6. "Temporal proximity:" You choose to give greater prominence and to infer more importance to associations of variables that have occurred more recently and to presume that earlier associations (or dissociations) should be "discounted."

\section{Evaluation of Results}

Once you have arrived at whatever stage in the research cycle you have chosen as your point of exit, your objective should be quite simple: make yourself the best possible critic of your own work. Anticipate all of the potential objections at each of the previous stages. Where possible, return and enter appropriate corrections. Since this is often impossible, given the numerous and irreversible "path dependencies" built into the research cycle, signal to your reader cum critic that you are aware of the defect and have tried your best not to be mislead by it or to magnify its impact. Above all, remind yourself right from the start that no research is perfect and all researchers make mistakes. Inscribe above your desk (or on your screensaver), the Latin phrase: Errare humanum est- "to make mistakes is to be human," would be my loose translation-and recognize that to be a human being studying human behaviour is to be doubly vulnerable to this maxim.

My overarching purpose in writing this chapter has been to help you to become your own best critic.

\section{Conclusion}

Social and political research is characterized by the diversity of its concepts, theories, designs — and logics. Only a few will work "around the clock" in Fig. 1 and conclude with empirically grounded inferences about causal relations among 


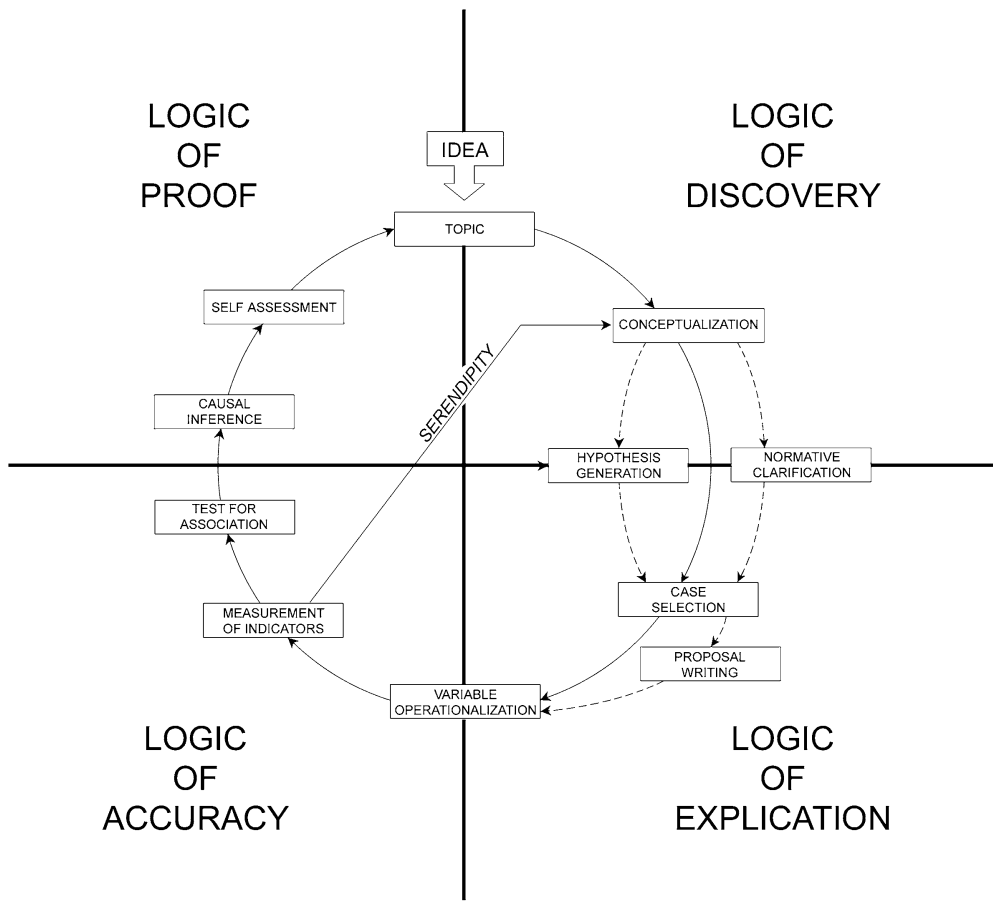

Fig. 3 The cycle of social and political research and its four logics

variables. Many will choose a topic for which this would be premature or inappropriate, given the existing state of his or her discipline or his or her purpose for selecting a particular topic. They may exit the cycle relatively early, sometime between 1 and 3 PM-hopefully, with an improved understanding of the generic relations involved and, possibly, with a more elaborate set of hypotheses for future research. Still others will be interested in drawing out the ethical and normative implications of these relationships, perhaps, by exploring analogies with previous experiences or prior philosophic assumptions. In Fig. 3, I have labelled this point of exit as "the Logic of Discovery," the idea being that those who take it will have made their original contribution by discovering empirical or normative relationships previously ignored or distorted by existing wisdom. The chapters in this volume by Zoe Bray. Alessandro Pizzorno, Sven Steinmo and Rainer Bauböck should be especially useful to those who choose to leave the cycle at this point.

From 3 to 6 PM, fewer social and political researchers will be leaving the cycle. ${ }^{1}$ Their distinctive contribution will have been to identify the apposite universe surrounding the topics selected, to select cases that represent specified distributions

\footnotetext{
1 Unfortunately, many of them will be so-called ABDs (all but dissertations) who come up with a design for a previously conceptualized piece of research, could have written a proposal and may even have given some thought about operationalizing its variables, but never managed to actually find the time, resources or energy to gather the relevant data-much less to write them up.
} 
of key variables and to have invented new ways of defining these variables and embedding them in more comprehensive theories. Most importantly, they will have carried further and in greater detail the existing conceptualization of the relationships surrounding their topic-hence, the notion that they have followed a "Logic of Explication." Donatella della Porta, Peter Mair, Christine Chwaszcza and Fritz Kratochwil have contributed chapters that should be of particular interest to them, but all of them presuppose that explication is not an end in itself, but only a necessary precondition for passing to the next stage which involves the specification of indicators and the gathering of data.

Many more social and political scientists will exit after 6 PM and before 9 PM. They will have produced research that is fundamentally descriptive in nature. Here, the preoccupation is with the validity of their measurements and the accuracy of their observations. They will have gone into the field-even if it is in their own backyard - and generated new data about social and political phenomena. They are also most likely to have contributed to the development of better instruments of observation and more reliable indicators. Mark Franklin's chapter deals primarily with this "Logic of Description."

The chapter by Adrienne Héritier is the one that comes closest to tackling headon the issues involved in the "Logic of Inference," although virtually all of the authors touch at least peripherally on the very controversial objective of making empirically grounded assumptions about causal relations in the political and social sciences. This may be where the ultimate payoff lies-and certainly where the highest disciplinary status is usually awarded-but only a select few make it to this stage in the cycle and even their conclusions are always contingent upon eventual replication by other scholars.

The reader should not be discouraged by this. To do original research on a topic you care about is an adventure. It can take you in different directions and end in different places. A lot will depend on your point of departure, but you will also be influenced at every turn by your professors and peers-not to mention the fads and fashions of your discipline. The most important thing is to be conscious and confident of the choices you will be making, and then to know when and where to exit from the cycle. Hopefully, this and the other essays in this volume will help you to make the voyage easier and, ultimately, more rewarding.

\section{Appendix: The 'Ideal' Research Proposal}

The following is a short outline of what I believe constitutes an ideal proposal, both for preparing to conduct social and political research and for applying to potential sponsors of this research. Needless to say, in the real world, no one actually writes "the" ideal proposal and all existing ones are based on some compromise tailored to fit the specificities of a particular project. It may be useful, however, to have this as a template against which to compare your own effort-if only to encourage you to think about what you did and did not put into your own proposal. 


\section{The Introduction}

A proposal has to be introduced. You have to shake hands with it before getting to know it better. This is much more important than many students realize because in many competitions, the reader will be evaluating a large number of proposals and may well not go much further that the first two paragraphs! You have to catch your reader-evaluator's attention with a compelling idea/theme/concept/method right at the beginning. Once (s)he has lost interest in what you propose to do, it will be very difficult to get it back.

1. The idea: What do you want to study?

Describe your topic/theme is a single sentence and follow with one or two short paragraphs "fleshing" out its basic properties: substance, location, key concepts, time period.

2. The reason: Why do you want to study it?

Explain where the idea came from and why you think it should be researched. Do not hesitate to relate it to normative concerns and/or events in the real political world, but somehow try to tie it into "the discipline" (whatever "it" is).

\section{The State of the Field}

Develop the relation of your topic/theme to the existing literature by explaining what we think we already know about it and how your work will improve upon or differ from this - in no more than two or three pages. Insert a mini-bibliography or biography by citing specific works and authors to illustrate the breadth, if not depth, of your preparation. If apposite, stress any unusual combination of literatures that you are making (that is known as "interdisciplinarity" and in some circles is very favourably received).

Complete your critical assessment of what has been written (and not written) on this topic with a statement of what your "posture" with regard to the existing wisdom is going to be. You have two options: (1) basically agree with what has been done and propose to extend it to a "missing case" or an "unlikely setting;" or (2) disagree with it and claim that you are prepared to offer and apply an alternative approach/theory/analytical test/methodological technique that is better. The first rests on a logic of confirmation: "no one has yet examined this specific event/case/ archive/time period(s) competently and, when I have, I am convinced that it will strengthen the existing theory;" the second involves something like a logic of disconfirmation: "I want to study an anomaly or puzzle that does not fit with what we think we know and, when I am finished, existing theories or methods will have to be revised, if not rejected outright."

Footnotes in this and subsequent parts of the text are a useful device for setting off parts of your argument and for demonstrating your scholarly vocation. 


\section{The Project Description}

In this middle section of ca. 7-8 pages, you should restate your initial idea in the form of a "model." The model does not have to be formal and explicit, but it should indicate both the external parameters: what you are taking for granted and proposing not to study, i.e. the universe of application and the conditions that you believe are shared or can be regarded as constant; and the internal "mechanisms:" what you regard as problematic and are going to study, i.e. the operative variables, the sequence of their occurrence and, especially, the hypothetical causal relations among them. The traditional format for doing this is via a set of "If ... then" hypotheses that, first, define a dependent variable (DV) or variables and, then, specify the independent variable (IV) or, more likely, variables that you believe "explain" variation in it. Needless to say, such a neat separation may not always be relevant and what is a dependent variable at time $\alpha$ may become an independent one at time $\beta$. Also, more sophisticated explanatory models may incorporate multiple intervening variables that can alter the anticipated relation between DVs and IVs. Even if you have no intention of testing a formal model with quantitative indicators, it is nevertheless a good idea to think in these terms when presenting a qualitative narrative argument because it can help you to better specify your argument for readers/evaluators-many of whom will be searching for evidence of "hard science" no matter what the topic.

This is also where you should indicate the temporal, as well as spatial and social, limits to your topic. Fashions in this regard have varied in the social sciences. At one time (and, again, with contemporary rational choice theory), priority was given to projects that claimed to be universalistic, i.e. valid for all times, places and persons. There is nothing "embarrassing" or "diminishing" about admitting that your topic is limited in any or all of these dimensions. Most "historical," "sociological," and "institutional" projects tend-implicitly or explicitly—to have such a restricted validity. And typologies (yours or those borrowed from others) tend to be especially useful in delimiting your universe of applicability and selecting cases for comparative analysis.

\section{The Research Design}

Here, you should take a few pages to outline two key aspects of any project: (1) its selection of cases/events/persons/processes/outcomes; and (2) its choice of methods for observation and inference. Why have you chosen a specific case or set of cases? What will you use to measure those variables that you consider "operational" and what will you use to test for the magnitude and significance of their relationship to each other?

Comparison is an especially valuable component of most social scientific designs. In its 'orthodox' version, this means a project with more than one unit of observation and, often, with either a sample of or all such units within a stipulated universe. If you choose this route, you should provide an indication of why you have selected these units and, if possible, why you have chosen either a "small N" or a "large N" design. NB It is possible to do "virtual' comparative research, and many 
doctoral students do this for quite practical and methodologically defensible reasons. By selecting only one case, the student can conceptualize it in a fashion that is potentially applicable to others - and can even introduce "contrasting" empirical material from these other cases while concentrating on the preferred one.

Statistical inference from quantitative data is also a valued asset in most branches of social science, but it is not indispensable. Everything depends on two factors: (1) the operationalizability of variables; and (2) the relationship between the number of cases and the number of variables. If your variables cannot be meaningfully transformed into cardinal or ordinal measures and if you intend to do a "deep description," i.e. one in which the number of observed conditions greatly outnumber the number of cases, then, you would be ill-advised to draw inferences from social statistics.

\section{The Feasibility of the Project}

This can be dropped from many proposals, but if there is a potential concern with the availability of data or the sensitivity to a particular topic, then, you should spend a page or less explaining how you might overcome these difficulties. Where you recognize the potentiality, then, you might say a few words about what you might do if data does not make itself available (it never does, but you can usually find it) or if the subjects involved do make themselves obstructive. An ideal design in this regard is like an artichoke-it has a heart that you are confident you can reach and exploit for your dissertation, but it also has a lot of external leaves that it would be nice to have but only to complement and enhance the core.

\section{The Payoff of the Project}

Normally, this is taken for granted. You write a thesis to obtain the doctoral degree and that is your "ticket" to an academic career. (If you do not want to be a teacher/ researcher in a university, you are probably ill-advised to even submit such a project). Beyond this, however, are two questions that are relevant to making a competitive judgment about your project: (1) why is the discipline(s) of ... (or the country of ...) going to be better off, if you compete this project successfully?; and (2) what is going to be the "generalizability" of whatever it is that you eventually discover? Obviously, a reader/evaluator that becomes convinced that this project is going to make a major (and, not just a marginal) contribution to political science or sociology is going to rank it higher-as (s)he is also likely to do, if convinced that it has a wide range of potential applications.

\section{The Bibliography}

For me, this seemingly banal coda at the end of the proposal plays a surprisingly important role. For one thing, it serves as a certification of what you have said about the state of the field and the breadth of your grasp of the topic. A project with an excessively summary or manifestly inappropriate bibliography loses a lot of credibility. For another thing, it serves as a demonstration of your "craftsmanship," 
i.e. your ability to follow a disciplinary set of rules in assembling sources and presenting them in a uniform fashion. A student who is sloppy, incomplete or inconsistent in the way that (s)he does a bibliography is probably going to do the same when writing the thesis. This, incidentally, also holds for footnotes, should you use them in the text.

Philippe C. Schmitter is currently professor emeritus at the European University Institute (EUI). He was a member of its Department of Political and Social Sciences from 1996 to 2004, after 10 years at Stanford University. He was educated at Dartmouth College, the National Autonomous University of Mexico, the University of Geneva and received his Ph.D. from the University of California, Berkeley. He was a professor for many years at the University of Chicago (1967-1982) and held visiting appointments at the University of Brazil in Rio de Janeiro, the Institute for the Integration of Latin America in Buenos Aires, Harvard University, the Universities of Geneva, Zürich, Paris and Mannheim, the Wissenschaftszentrum in Berlin, the Institut d'Etudes Politiques in Paris, the Fundación Juan March in Madrid and the Instituto de Ciencias Sociais in Lisbon. Before coming to Stanford in 1986, he spent the previous 4 years as a professor at the EUI. Currently, he has been a recurrent visiting professor at the Central European University in Budapest, at the Istituto delle Scienze Humanistiche of the Scuola Normale di Superiore in Florence and the University of Siena. He has been the recipient of the Johan Skytte Prize of the University of Uppsala, the Mattei Dogan Prize of the ECPR and the Lifetime Achievement Award of the European Studies Association. 